Z. klin. Chem. u. klin. Biochem.

7. Jg., S. 346-349, Juli 1969

\title{
Nachweis von Paracetamol im Urin nach Einnahme von therapeutischen Mengen phenacetinhaltiger Analgetika
}

\author{
Von S. GOENECHEA \\ Aus dem Institut für Gerichtliche Medizin der Universität Bonn (Direktor: Prof. Dr. H. Elbel) ·
}

(Eingegangen am 3. Februar 1969)

Es wird eine einfache Methode zum dünnschichtchromatographischen Nachweis von Paracetamol im Urin angegeben. Der Urin wird bei einem $\mathrm{pH}$ von 3 direkt mit Chloroform extrahiert, der Harnextrakt chromatographiert und die Platte mit Silbernitratreagenz besprüht. Das Verfahren ist an Urinproben mehrerer Versuchspersonen angewandt worden. Ferner wurden die Urinextrakte nach BRopie und AXELROD spektralphotometrisch untersucht.

The demonstration of paracetamol in the urine following the ingestion of therapeutic doses of phenacetin-containing analgesics

A simple method is described for the detection of urinary paracetamol by thin layer chromatography. The urine is extracted with chloroform at $\mathrm{pH} 3$, the extract is chromatographed and the plate is sprayed with silver nitrate reagent. The method was applied to urine samples from several experimental persons. The urine extracts were also studied by the spectrophotometric method of BRODIE and AxELROD.

Eine große Zahl von Kombinationspräparaten der Gruppe der Analgetika enthält Phenacetin. (I). Diese Pharmaka finden breite Anwendung. Über den Mißbrauch von phenacetinhaltigen Präparaten und seine Folgen ist mehrfach berichtet worden (1-5). Bei den Kraftfahrern stehen die Analgetika im Gebrauch weit an der Spitze (6,7). Vielfach ist es deshalb im Zusammenhang mit einer verkehrsmedizinischen Beurteilung eines Kraftfahrers wichtig, die Einnahme phenacetinhaltiger Arzneimittel nachzuweisen.

Etwa $60-80 \%$ des verabreichten Phenacetins (I) werden als Paracetamol (II) überwiegend konjugiert im Harn wiedergefunden, während nur etwa $0,2 \%$ der eingenommenen Phenacetinmenge unverändert ausgeschieden wird $(8,9)$. Ferner treten $0,1-0,2 \%$ der Dosis als $p$-Äthoxyanilin ( $p$-Phenetidin) (V) im Urin auf (Abb. 1). JAGENBURg und Toczko (10) fanden außerdem, daß etwa $2 \%$ des verabreichten Phenacetins als S-(1-Acetamido-4-hydroxyphenyl)-cystein im Urin auftritt.

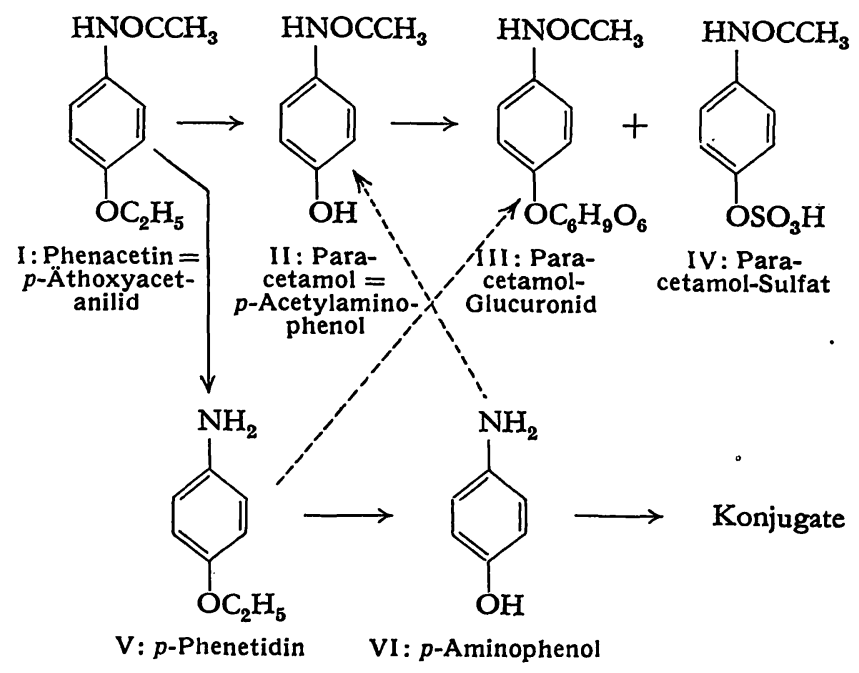

Abb. 1. Biochemischer Umbau von Phenacetin im Organismus (11)
Nach Einnahme von Phenacetin findet man im Harn nach saurer Hydrolyse $p$-Aminophenol (VI). Diese Substanz entsteht sowohl aus dem freien Paracetamol durch Abspaltung der Acetylgruppe - als auch aus den Konjugaten - durch zusätzliche Abspaltung des Sulfat- oder Glucuronid-Restes. Die bisher angegebenen Nachweismethoden $(8,9,12-15)$ sind für die Routine zu umständlich und eignen sich nur zur Durchführung einer gezielten Untersuchung.

Oft fehlen dem Toxikologen aber Angaben über die Art des eingenommenen Medikamentes oder dieselben entsprechen nicht der Wahrheit (16). Es muß deshalb eine einfache Methode gefunden werden, die - routinemäßig ausgeführt - nicht nur auf den Nachweis der Stoffwechselprodukte des Phenacetins bzw. ihres $\mathrm{Hy}$ drolyseproduktes abgestellt ist, sondern auch bei der Untersuchung auf andere Pharmaka angewandt werden kann. Darüber hinaus soll es auch möglich sein, nicht nur bei Vergiftungen oder bei chronischem Abusus, sondern auch bei therapeutischem Gebrauch eine Phenacetineinnahme nachzuweisen.

Im allgemeinen geht man so vor, $\mathrm{da} B$ man die Harnproben ansäuert und zunächst mit Äther oder Chloroform ausschüttelt $(6,14,16,17,18,19)$; der Verdampfungsrückstand wird anschließend chromatographisch untersucht.

Phenacetin, Paracetamol sowie ihr Hydrolyseprodukt $p$-Aminophenol können auf Fluoreszenzschichten (12, 19, 20) oder nach Besprühen des Chromatogramms mit ammoniakalischer Fluoresceinlösung (21) unter UVLicht erkannt werden. Auch Dragendorff (22) und Ehrlich-Reagens (22) sowie Chlor-Benzidin (23), Dichromat-Schwefelsäure (23), Kaliumpermanganat/Kaliumcarbonat und Jodatmosphäre (24) u. a. m. sind verwendet worden. Alle diese Nachweismethoden sind wenig spezifisch; viele andere Arzneimittel und körpereigene Stoffe können dabei sichtbar gemacht werden. Auch mit Eisen[III]-chlorid lassen sich außer Phe- 
nacetin und Paracetamol $(25,26)$ andere Arzneistoffe anfärben $(27,28)$.

Größere Spezifität besitzt das Silbernitratreagenz (25, 26); mit diesem Sprühmittel können 0,2 $\mu \mathrm{g}$ Paracetamol nachgewiesen werden. Phenacetin wird mit der Silbernitratlösung nicht angefärbt; diese Substanz wird jedoch nur in sehr geringen Mengen unverändert ausgeschieden, so daß der Nachweis einer Phenacetineinnahme im Urin am besten über das Stoffwechselprodukt - Paracetamol (II) - gelingen wird.

So konnten wir in den bei saurer Reaktion erhaltenen Chloroformextrakten von Urinproben mehrerer Versuchspersonen Paracetamol nachweisen, nachdem die Probanden therapeutische Einzeldosen eines phenacetinhaltigen Kombinationspräparates eingenommen hatten.

Paracetamol wurde außerdem in den bei saurer Reaktion erhaltenen Harnextrakten nach dem Verfahren von Brodie und AXELROD (8) spektralphotometrisch bestimmt.

\section{Arbeitsmethodik}

\section{Extraktion des Urins}

$25 \mathrm{~m} /$ Harn wurden mit der dreifachen Menge einer Pufferlösung vom $\mathrm{pH} 3$ (Puffer-Titrisol, E. Merck, Darmstadt) versetzt und dreimal mit Chloroform extrahiert. Die vereinigten Chloroformlösungen wurden mit demselben Lösungsmittel auf $200 \mathrm{ml}$ aufgefüllt und danach in zwei gleiche Portionen aufgeteilt; die eine Hälfte wurde nach dem Abdampfen des Lösungsmittels dünnschichtchromatographisch untersucht, während die andere Hälfte für die quantitative Bestimmung verwendet wurde.

\section{Dünnschichtchromatographie}

Es wurden Platten von $200 \times 200 \mathrm{~mm}$ verwendet, die mit Kieselgel G nach dem Standardverfahren von STAHL (29) beschichtet waren. Die Chromatographie erfolgte nach dem Verfahren der zweidimensionalen Trennung (29).

\section{Fließmittel}

Richtung 1: Chlotoform/Aceton $(90: 10, \nabla / \nabla)(29)$

Richtung 2: Chloroform/Benzol/Aceton (65:5:30, $v / \mathrm{v})$.

Nach der Entwicklung des Chromatogramms in Richtung 1 wurde die Platte mit Hilfe eines Haartrockners getrocknet, die Vergleichslösung aufgetragen und dann in Richtung 2 erneut chromatographiert. Laufstrecke: jeweils etwa $16 \mathrm{~cm}$.

\section{Nacbweisreagens}

10proz. wäßr. Silbernitratlösung $(25,30)$. Flecke treten bei Raumtemperatur nach 45-20 Min. - je nach Menge - auf.

\section{Spektralphotometrische Bestimmung}

Der nach Destillation des Chloroforms erhaltene Rückstand wuirde mit $5 \mathrm{ml} 1 \mathrm{~N}$ Salzsäure versetzt und abfiltriert; nach $1 \mathrm{stdg}$. Behandlung des Filtrates im siedenden Wasserbad wurde die quantitative Bestimmung nach dem Verfahren von BrodIE und AXELroD (8) durchgeführt. Die Extinktion wurde in $10 \mathrm{~mm}-$ Halbmikroküvetten bei $570 \mathrm{~nm}$ gegen einen gleichbehandelten Bilanzansatz gemessen, in dem die Analyselösung durch ein gleiches Volumen dest. Wasser ersetzt worden war.

Zur Auswertung stellt man mit Hilfe von Standardlösungen entsprechend 20 bis $1400 \mu \mathrm{g}$ Paracetamol - , die nach vorstehender Vorschrift behandelt wurden, eine Eichkurve auf. Die Messung erfolgte mit einem Unicam-Leitz-Spektralphotometer Modell SP $800 \mathrm{~B}$.

\section{Ergebnisse und Diskussion}

Der Nachweis und die Bestimmung der Stoffwechselprodukte des Phenacetin ist mehrfach in Sammelurinen von 8 bzw. 24 Stunden $(8,12)$ durchgeführt worden. WeLCH und ConNey (15) bestimmten die 2stdg. Ausscheidung von Paracetamol. Dubach (13) führte seine Untersuchungen sowohl an Spontanurin als auch an Sammelurinen durch.

In der Regel stehen dem Toxikologen keine Sammelurine zur Verfügung. Deshalb wurde von uns nur Spontanurin untersucht; wir haben die Probanden veranlaßt, ihrem normalen Bedürfnis entsprechend die vier der Einnahme des Analgetikums folgenden Harne einzeln zu asservieren.

Für unsere Untersuchungen stellten sich sechs Personen zur Verfügung, zwei weibliche und vier männliche. Ihr Alter lag zwischen 22 und 35 Jahren. Als phenacetinhaltige Analgetika wählten wir die Kombinationspräparate Quadronal und Dolviran, denn sowohl der chromatographische Paracetamolnachweis als auch seine photometrische Bestimmung sollten in Gegenwart von Stoffwechselprodukten anderer Pharmaka erfolgen, so wie es in der Praxis oft der Fall ist. Jede Versuchsperson führte je einen Versuch mit jedem Kombinationspräparat durch. Die Einnahme von jeweils zwei Tabletten erfolgte morgens nach dem Frühstück.

1 Dragee Quadronal (Asta, Brackwede) enthält: Phenyldimethylpyrazolon $100 \mathrm{mg}$, Phenacetin $100 \mathrm{mg}$, Lactylphenetidin $1,5 \mathrm{mg}$ und Coffeinum anhydr. $50 \mathrm{mg}$.

1 Tablette Dolviran (Bayer) enthält: Aspirin $200 \mathrm{mg}$, Phenacetin $200 \mathrm{mg}$, Codein. phosphoric. $10 \mathrm{mg}$, Coffeinum anhydr. $50 \mathrm{mg}$, Luminal $25 \mathrm{mg}$.

Ungeachtet des gelassenen Harnvolumens wurden zur Untersuchung jeweils $25 \mathrm{~m} l$ Urin bei einem $\mathrm{pH}$ von 3 mit Chloroform extrahiert. Die Hälfte des Chloroformextraktes (entsprechend $12,5 \mathrm{ml}$ Urin) wurde dünnschichtchromatographisch auf Paracetamol untersucht, während die andere Hälfte zur spektralphotometrischen Bestimmung des Paracetamols diente. Die Dünnschichtchromatographie wurde nach dem Verfahren der zweidimensionalen Trennung durchgeführt, da sich bei der eindimensionalen Chromatographie erhebliche Störungen des Paracetamolnachweises durch die Ballaststoffe des Harnextraktes zeigten.

Bei allen durchgeführten Versuchen konnte Paracetamol schon im ersten Urin (1,5-4 Stdn. nach der Einnahme) festgestellt werden. Im vierten Urin (8-9 Stdn. nach der Einnahme) war der Paracetamolnachweis auch im Quadronalversuch - entsprechend $200 \mathrm{mg}$ Phenacetin - positiv. Die Ergebnisse der Chromatographie der Harnextrakte sind in den Tabelle 1 und 2 wiedergegeben.

Adalin und Bromural sowie die Hydantoinderivate Revertonal weiß und Revertonal braun werden ebenfälls mit dem Silbernitratreagens angefärbt (31); mit den hier verwendeten Fließmitteln zeigt das Paracetamol einen $R_{\mathrm{F}}$-Wert, der weit unter dem der anderen mit Silbernitratreagenz anfärbbaren Substanzen liegt, so daß die Unterscheidung keinerlei Schwierigkeiten bereitet. Außerdem zeigten frühere Untersuchungen, daß Adalin und Bromural in therapeutischen Mengen eingenommen im Urin nicht nachgewiesen werden können (30); somit 
Tab. 1

DC-Nachweis von Paracetamol im Harn nach Einnahme von 2 Dragees Quadronal (entsprechend $200 \mathrm{mg}$ Phenacetin)

\begin{tabular}{|c|c|c|c|c|}
\hline Versuch & Harnprobe & $\begin{array}{c}\text { Harn- } \\
\text { volumen } \\
\text { (Gesamt) }\end{array}$ & $\begin{array}{c}\text { Stunden } \\
\text { nach } \\
\text { Einnahme }\end{array}$ & $\begin{array}{l}\text { Para- } \\
\text { cetamol- } \\
\text { nachweis }\end{array}$ \\
\hline 1 & $\begin{array}{l}\text { I } \\
\text { II } \\
\text { III } \\
\text { IV }\end{array}$ & $\begin{array}{l}1455^{\circ} \mathrm{ml} \\
175 \mathrm{ml} \\
250 \mathrm{ml} \\
165 \mathrm{ml}\end{array}$ & $\begin{array}{l}11 / 2 \\
4^{3} / 4 \\
6^{2} 1 / 2 \\
8^{1} 1 / 2\end{array}$ & $\begin{array}{l}+ \\
+ \\
+ \\
+\end{array}$ \\
\hline 2 & $\begin{array}{l}\text { I } \\
\text { II } \\
\text { II }\end{array}$ & $\begin{array}{r}90 \mathrm{ml} \\
75 \mathrm{ml} \\
135 \mathrm{ml}\end{array}$ & $\begin{array}{l}2 \\
5 \\
8 \\
-\end{array}$ & $\begin{array}{l}+ \\
+ \\
+\end{array}$ \\
\hline 3 & $\begin{array}{l}\text { I } \\
\text { IIII } \\
\text { IV }\end{array}$ & $\begin{array}{r}70 \mathrm{ml} \\
265 \mathrm{ml} \\
325 \mathrm{ml} \\
300 \mathrm{ml}\end{array}$ & $\begin{array}{l}2^{1 / 1} \\
5^{3 / 4} \\
7 \\
8^{1 / 3}\end{array}$ & $\begin{array}{l}+ \\
\stackrel{+}{+} \\
(+) \\
(+)\end{array}$ \\
\hline 4 & $\begin{array}{l}\text { I } \\
\text { II } \\
\text { III } \\
\text { IV }\end{array}$ & $\begin{array}{l}45 \mathrm{ml} \\
55 \mathrm{ml} \\
75 \mathrm{ml} \\
60 \mathrm{ml}\end{array}$ & $\begin{array}{l}11 / 2 \\
2^{3} / 4 \\
4^{1} 1 / 2 \\
6^{1} / 4\end{array}$ & $\begin{array}{l}+ \\
+ \\
+ \\
+\end{array}$ \\
\hline 5 & $\begin{array}{l}\text { I } \\
\text { II } \\
\text { III } \\
\text { IV }\end{array}$ & $\begin{array}{l}245 \mathrm{ml} \\
130 \mathrm{ml} \\
325 \mathrm{ml} \\
180 \mathrm{ml}\end{array}$ & $\begin{array}{l}3 \\
4^{3} / 4 \\
7 / 4 \\
8^{1} / 4\end{array}$ & $\begin{array}{l}+ \\
+ \\
\stackrel{+}{+} \\
(+)\end{array}$ \\
\hline 6 & $\begin{array}{l}\mathrm{I} \\
\text { II } \\
\text { III } \\
\text { riv }\end{array}$ & $\begin{array}{r}180 \mathrm{ml} \\
240 \mathrm{ml} \\
70 \mathrm{ml} \\
180 \mathrm{ml}\end{array}$ & $\begin{array}{l}11 / 2 \\
31 / 2 \\
5 \\
73 / 4\end{array}$ & $\begin{array}{c}(+) \\
(+) \\
+ \\
+\end{array}$ \\
\hline
\end{tabular}

Tab. 2

DC-Nachweis von Paracetamol im Harn nach Einnahme von 2 Tabletten Dolviran (entsprechend $400 \mathrm{mg}$ Phenacetin)

\begin{tabular}{|c|c|c|c|c|}
\hline Versuch & Harnprobe & $\begin{array}{c}\text { Harn- } \\
\text { volumen } \\
\text { (Gesamt) }\end{array}$ & $\begin{array}{l}\text { Stunden } \\
\text { nach } \\
\text { Einnahme }\end{array}$ & $\begin{array}{l}\text { Para- } \\
\text { cetamol- } \\
\text { nachweis }\end{array}$ \\
\hline 1 & $\begin{array}{l}\text { I } \\
\text { II } \\
\text { III } \\
\text { IV }\end{array}$ & $\begin{array}{l}135 \mathrm{ml} \\
185 \mathrm{ml} \\
205 \mathrm{ml} \\
235 \mathrm{ml}\end{array}$ & $\begin{array}{c}4 \\
6^{1} / 4 \\
9 ! \\
10^{1} / 2\end{array}$ & $\begin{array}{l}+ \\
+ \\
+ \\
+\end{array}$ \\
\hline 2 & $\begin{array}{l}\text { I } \\
\text { II } \\
\text { III }\end{array}$ & $\begin{array}{r}41 \mathrm{ml} \\
210 \mathrm{ml} \\
215 \mathrm{ml} \\
75 \mathrm{ml}\end{array}$ & $\begin{array}{l}2 \\
4^{1} / 2 \\
61 / 2 \\
8^{1} 1 / 2\end{array}$ & $\begin{array}{l}+ \\
+ \\
+ \\
+\end{array}$ \\
\hline 3 & $\begin{array}{l}\text { I } \\
\text { II } \\
\text { III } \\
\text { IV }\end{array}$ & $\begin{array}{l}115 \mathrm{ml} . \\
225 \mathrm{ml} \\
240 \mathrm{ml} \\
255 \mathrm{ml}\end{array}$ & $\begin{array}{l}2 \\
4 \\
6 \\
81 / 4\end{array}$ & $\begin{array}{l}+ \\
+ \\
+ \\
+\end{array}$ \\
\hline 4 & $\begin{array}{l}\text { I } \\
\text { III } \\
\text { IV }\end{array}$ & $\begin{array}{l}80 \mathrm{ml} \\
63 \mathrm{ml} \\
60 \mathrm{ml} \\
68 \mathrm{ml}\end{array}$ & $\begin{array}{r}2^{3} 3 / 4 \\
5^{2} / 2 \\
71 / 2 \\
10^{1 / 3}\end{array}$ & $\begin{array}{l}+ \\
+ \\
+ \\
+\end{array}$ \\
\hline 5 & $\begin{array}{l}\text { I } \\
\text { II } \\
\text { III }\end{array}$ & $\begin{array}{l}175 \mathrm{ml} \\
125 \mathrm{ml} \\
135 \mathrm{ml} \\
105 \mathrm{ml}\end{array}$ & $\begin{array}{l}3 \\
5^{a} / 4 \\
71 / 2 \\
9\end{array}$ & $\begin{array}{l}+ \\
+ \\
+ \\
+\end{array}$ \\
\hline 6 & $\begin{array}{l}\text { I } \\
\text { II } \\
\text { III }\end{array}$ & $\begin{array}{r}115 \mathrm{ml} \\
270 \mathrm{ml} \\
115 \mathrm{ml} \\
80 \mathrm{ml}\end{array}$ & $\begin{array}{l}11 / 2 \\
3^{12} \\
4^{1} / 2 \\
6\end{array}$ & $\begin{array}{l}+ \\
+ \\
+\end{array}$ \\
\hline
\end{tabular}

kommt eine Störung des Paracetamolnachweises durch diese Verbindungen nicht in Betracht.

Unsere Untersuchungen haben außerdem ergeben, daß $p$-Aminophenol auch mit dem Silbernitratreagenz angefärbt wird. Diese Substanz erscheint jedoch sofort beim Besprühen des Chromatogramms als grauschwarzer Fleck; der Paracetamolfleck dagegen tritt erst nach 10-40 Min. - je nach Menge - auf. p-Aminophenol weist ferner mit den hier verwendeten Fließmitteln einen höheren $R_{\mathrm{F}}$-Wert als Paracetamol auf. Beide Stoffe lassen sich gut voneinander unterscheiden.

Die Chloroformextrakte wurden zur weiteren Sicherung und Spezifizierung des DC-Befundes nach dem Verfahren von Brodie und AXELroD (8) spektralphotometrisch untersucht. In den Rückständen der ersten zwei
Urine nach der Einnahme des Analgetikums fand man erhebliche Mengen Paracetamol; die Bestimmung mußte deshalb mehrmals mit 1 bzw. $5 \mathrm{~m} l$ Urin anstatt mit $12,5 \mathrm{~m} l$ durchgeführt werden. Die bei allen Versuchen durchschnittlich festgestellte Paracetamolmenge betrug in den ersten $z$ wei Urinen $\left(11 / 2-6^{1} / 4\right.$ Stdn. nach der Einnahme) zusammen etwa $11 \%$ der eingenommenen Dosis. In den folgenden Urinen nahmen die Paracetamolmengen bei den meisten Probanden rasch ab und im vierten Urin war die quantitative Erfassung des Paracetamols - in $12,5 \mathrm{ml}$ Urin - oft nicht möglich, obwohl der dünnschichtchromatographische Nachweis eindeutig das Vorliegen des Stoffwechselproduktes von Phenacetin zeigte. In solchen Fällen konnte jedoch im Chloroformextrakt des Gesamturins Paracetamol spektralphotometrisch bestimmt werden. Eine Ausnahme vom allgemeinen Verhalten zeigte die Versuchsperson 5 bei dem Versuch mit Quadronal (Tab. 1). Bei der Chromatographie der Harnextrakte 1 und 2 war der Paracetamolfleck schwach. Die quantitative Bestimmung in den ersten zwei Urinen ergab nur geringe Mengen Paracetamol, während in der 3. und 4. Urinprobe $8-9 \%$ der verabreichten Dosis festgestellt werden konnten.

WeLCH und ConNey (15) und später BüCH, Häuser, PFLËGER und RüDIGER (12) fanden, daß in den ersten 8 Stdn. nach der Einnahme von Phenacetin $40-60 \%$ der Dosis ausgeschieden wird. Sie bestimmen dabei das freie sowie das konjugierte Paracetamol. Bei dem von uns angewandten Verfahren wird ein Teil des Paracetamols nicht erfaßt, da hier keine saure Hydrolyse vorgenommen wird; mit unseren Versuchen sollte festgestellt werden, ob eine Einnahme therapeutischer Mengen Phenacetins auch dann nachgewiesen werden kann, wenn man die Urinprobe in bekannter Weise direkt ausschüttelt.

Bei der direkten Extraktion des Urins mit Chloroform bei einem $\mathrm{pH}$ von 3 wird offensichtlich ein Teil des als Konjugat vorliegenden Paracetamols in die organische Phase überführt und somit quantitativ mitbestimmt, denn die von uns festgestellten Ausscheidungsquoten liegen schon in den ersten Stunden erheblich höher als diejenigen, die von Brodie und Axelrod (8) für das freie Paracetamol angegeben werden; sie fanden in 24-Stundenurin nur 3,5\% des applizierten Phenacetins als freies Paracetamol im Urin.

Die direkte Extraktion des Harnes beim pH 3 und die anschließende Chromatographie des Chloroformextráktes mit der Silbernitratlösung als Nachweisreagenz stellen ein einfaches und spezifisches Nachweisverfahren für Paracetamol dar. Der erhaltene Chloroformextrakt kann auch für die chromatographische Vorprobe auf andere Arzneistoffe (Barbiturate, Bromureide u. a. m.) verwendet werden. Der bei saurem $\mathrm{pH}$ mit Chloroform extrahierte Urin kann außerdem in der üblichen Weise einer fraktionierenden Extraktion im sodaalkalischen und natriumbicarbonatalkalischen Medium unterworfen und die erhaltenen Extraktrückstände können auf basische Arzneistoffe untersucht werden. 
Obwohl nach unserem Verfahren vorwiegend das freie, nichtkonjugierte Paracetamol erfaßt wird, kann eine einmalige Einnahme eines phenacetinhaltigen Analgetikums entsprechend $200 \mathrm{mg}$ Phenacetin nach etwa $8 \mathrm{Stdn}$. in $12-13 \mathrm{ml}$ Urin nachgewiesen werden; es ist somit nicht erforderlich, für den qualitativen Nachweis einer Phenacetineinnahme eine Hydrolyse des Harnes vorzunehmen. Außerdem besteht auch die Möglichkeit, das als Konjugat vorliegende Paracetamol quantitativ zu bestimmen, indem man den extrahierten Harn zusätzlich einer sauren Hydrolyse unterwirft; damit werden Paracetamolsulfat und Paracetamolglucuronid in $p$-Aminophenol überführt und dieses kann in der üblichen Weise quantitativ bestimmt werden.

\section{Literatur}

1. Gsell, O., U. C. Dubach und U. Raillard-Peucker, Disch. med. Wschr. 93, 101 (1968). - 2. Gsell, O. und M. STrobel, Schweiz. med. Wschr. 97, 661 (1967). - 3. KIrLholz, P. und R. Battegay, Praxis (Bern) 53, 447 (1964). - 4. Marti, Th., Praxis (Bern) 53, 442 (1964). - 5. Scheidegger, S., Arch. Toxikol. 19, 19 (1961). - 6. Pribilia, O., Zbl. Verkehrs-Med., VerkehrsPsych., Luft- u. Raumfahrtmedizin 2, 3 (1965). - 7. WAGNER, H.-J., Arzneimittel-Forsch., Aulendorf 11, 992 (1961). - 8. Brodie, B. B. und J. Axelrod, J. Pharmacol. Exper. Therap. Baltimore 97, 58 (1949). - 9. SMrth, J. N. und R. T. Williams, Biochem. J. 44, 239 (1949). - 10. JagenBurg, O. R. und K. Toczko., Biochem. J. 92, 639 (1964). - 11. Stewart, C. P. und A. Stolman, Toxicology. Vol. I. Academic Press. New YorkLondon (1960). - 12. Büch, H., H. HäUSER, K. PFLEGER und W. Rüdiger, diese Z. 4, 288 (1966). - 13. Dubach, U. C., Dtsch. med. Wschr. 92, 211 (1967). - 14. OBersteg, J. I. und J. BÄUMLER, Schweiz. med. Wschr. 97, 1039 (1967). - 15. WELCH, R. M. und A. H. Conney, Clin. Chem. (New York) 11, 1064 (1965). 16. Schmidr, G., J. Bösche und H. Keding, Aktuelle Probleme der Verkehrsmedizin 3, 45 (1966). - 17. MachatA, G. und W.
Krsser, Arch. Toxikol. 19, 327 (1962). - 18. Mohrschulz, W., Arch. Pharmazie, Berlin 289, 508 (1956). - 19. Büch, H., G. KARAChristianidis, K. Pflegger, W. Forth und D. Wörner, Arch. Toxikol. 21, 216 (1966). - 20. Büch, H., K. Pfleger und W. RÜDIGER, diese Z. 5, 110 (1967). - 21. BONNICHSEN, R., A. C. MAEhly und S. NordLänder, J. Chromatog. 3, 190 (1960). 22. Schmidr, G., Dtsch. Zschr. gerichtl. Med. 49, 259 (1959). 23. Dressler, A., Dtsch. Zschr. gerichtl. Med. 50, 457 (1960). 24. Gänshirt, H., Arch. Pharmazie Berlin 296, 73 (1963). 25. Goenechea, S., J. Chromatog. 38, 145 (1968). - 26. ShIbasaki, J., T. Koizumi, T. Tanaka und N. Nakatomi, Chem. Pharm. Bull. (Japan) 16, 1726 (1968). - 27. Goenechea, S., Mitteilungsblatt GDCh-Fachgr. Lebensmittelchem. gerichtl. Chem. 22, 340 (1968). - 28. GoenecheA, S., Arch. Toxikol. (im Druck). 29. StAHL, E., Dünnschichtchromatographie. 2. Auflage. SpringerVerlag. Heidelberg-Berlin-New York (1967). - 30. GoenECHEA, S. und R. ButtleR, Z. analyt. Chem. 239, 389 (1968). - 31. Paulus, W., S. Goenechea und R. Buttler, ArzneimittelForsch., Aulendorf 19, 388 (1969).
Dr. S. Goenechea 53 Bonn Stiftsplatz 12 